\title{
Head Trauma in Female Professional Wrestlers
}

\author{
Jun NOMOTO, Yoshikatsu SEIKI, Masaaki NEMOTO, Hiroshi TAKAHASHI*, \\ Hiroshi TERASHIMA*, Kyosuke YOKOTA, Kosuke KONDO, \\ Toshiyuki KANO, Syozo GOTO, and Nobuo SUGO
}

\begin{abstract}
Departments of Neurosurgery and *Orthopedic Surgery, Toho University, Omori Medical Center, Tokyo
\end{abstract}

\begin{abstract}
The clinical characteristics of head trauma were evaluated in 18 wrestlers belonging to a female professional wrestling organization, 13 regular members and five trainees aged 15-34 years. Medical examinations for head trauma were performed in all wrestlers, and wrestlers treated at our emergency outpatient department were clinically evaluated. In addition, the relationships of head trauma with duration of the wrestling career of 1-16 years (mean 8 years) in the regular members, and less than 1 year in the five trainees, and body mass index (BMI) of 21.0-32.0 in the 16 subjects, excluding two trainees, was evaluated. Chronic symptoms were noted in four of the 18 wrestlers with long wrestling careers (16 years in 1, 13 years in 1, and 5 years in 2). Three wrestlers with symptoms immediately after head trauma showed recurrent retrograde amnesia and had low BMI (21.6, 21.6, and 23.1). Five wrestlers were treated at our emergency outpatient clinic, three required hospitalization and two showed intracranial traumatic changes on computed tomography (acute subdural hematoma in 1 and diffuse brain swelling in 1). Head trauma in female professional wrestlers is associated with longer wrestling career and low BMI. Periodic medical examinations are recommended to monitor for signs of head trauma.
\end{abstract}

Key words: head trauma, medical check, body mass index, female, wrestling

\section{Introduction}

Head trauma suffered during sports activities is well known, 1,2,6,7,13,14,16,21,23-25) and incidents associated with snowboarding have increased recently with the rise in the popularity of the sport. ${ }^{19)}$ Professional wrestling in Japan has lead to the deaths of two female and one male wrestlers due to head trauma since $1997,{ }^{20}$ ) but these incidents have not been adequately evaluated. In particular, head trauma in female professional wrestlers remains uncharacterized.

This study investigated the clinical characteristics of head trauma in female professional wrestlers.

\section{Materials and Methods}

Eighteen wrestlers belonging to a female professional wrestling organization were examined, 13 regular members and five trainees aged 15-34 years (mean 24.2 years), who participated in 817 matches from November 2000 to December 2001 (Table 1).

Received July 3, 2006; Accepted December 12, 2006
The regular members had been professional wrestlers for 1-16 years (mean 8 years) and the five trainees for less than 1 year. Height and body weight were measured in 16 members, excluding two trainees, as 154-168 cm (mean $162.9 \mathrm{~cm}$ ) and 53-82 $\mathrm{kg}$ (mean $65.1 \mathrm{~kg}$ ), with body mass index (BMI) (normal 20-24) of 21.0-32.0 (mean 24.5).

Each wrestler was questioned about perceived chronic symptoms and symptoms which had developed immediately after head trauma, and was examined neurologically. Computed tomography (CT) was performed in the 16 wrestlers who consented. Clinical findings were also evaluated in five wrestlers treated for head trauma at the emergency outpatient clinic of our hospital during the investigation period. The relationships between clinical findings of head trauma and the career duration as a professional wrestler and BMI were evaluated.

\section{Results}

Four of the 18 wrestlers were aware of chronic symptoms (Table 1). Their professional career was 16 years in one, 13 years in one, and 5 years in two. 
Table 1 Summary of medical examinations

\begin{tabular}{|c|c|c|c|c|c|c|}
\hline Case No. & $\begin{array}{l}\text { Age (year)/ } \\
\text { Career }\end{array}$ & $\begin{array}{l}\text { Height }(\mathrm{cm}) / \\
\text { Weight }(\mathrm{kg})\end{array}$ & BMI & $\begin{array}{l}\text { Chronic } \\
\text { symptoms }\end{array}$ & Symptoms after head trauma & CT findings \\
\hline 1 & $34 / 16$ & $168 / 76$ & 26.9 & dizziness & n.d. & n.p. \\
\hline 3 & $31 / 13$ & $164 / 63$ & 23.4 & headache & n.d. & n.p. \\
\hline 4 & $30 / 12$ & $160 / 82$ & 32 & n.d. & n.d. & n.p. \\
\hline 5 & $29 / 12$ & $162 / 70$ & 26.7 & n.d. & n.d. & - \\
\hline 8 & $23 / 5$ & $162 / 58$ & 22.1 & headache & n.d. & n.p. \\
\hline 9 & $23 / 5$ & $167 / 78$ & 28 & n.d. & n.d. & n.p. \\
\hline 10 & $21 / 5$ & $157 / 60$ & 24.3 & n.d. & n.d. & n.p. \\
\hline 11 & $26 / 5$ & $164 / 58$ & 21.6 & n.d. & retrograde amnesia & n.p. \\
\hline 12 & $22 / 5$ & $164 / 62$ & 23.1 & n.d. & $\begin{array}{l}\text { retrograde amnesia, } \\
\text { transient unconsciousness }\end{array}$ & n.p. \\
\hline 16 & $17 / 0^{*}$ & unknown & unknown & n.d. & n.d. & n.p. \\
\hline 17 & $16 / 0^{*}$ & $154 / 53$ & 22.3 & n.d. & n.d. & n.p. \\
\hline 18 & $15 / 0^{*}$ & $155 / 57$ & 23.7 & n.d. & n.d. & n.p. \\
\hline
\end{tabular}

*Career 0: trainee (Cases 14-18). BMI: body mass index, CT: computed tomography, n.d.: not described, n.p.: no problem, - : not performed.

Table 2 Summary of examinations at the emergency outpatient clinic

\begin{tabular}{cllrr}
\hline Case No. & \multicolumn{1}{c}{ Symptoms } & CT findings & MR imaging findings & Admission (day) \\
\hline 2 & headache, laceration & n.p. & - \\
4 & diplopia & n.p. & n.p. \\
9 & headache, nausea, numbness of hands & brain swelling & - & - \\
11 & headache, transient unconsciousness & n.p. & ASDH \\
14 & headache, nausea & ASDH & 10 \\
\hline
\end{tabular}

ASDH: acute subdural hematoma, CT: computed tomography, MR: magnetic resonance, n.p.: no problem, 一: not performed.

However, no particular trend was noted in the BMI of these wrestlers.

Three wrestlers reported symptoms immediately after head trauma, and retrograde amnesia was observed in all three wrestlers (Table 1). All three wrestlers had had two or more episodes of retrograde amnesia, and one had also experienced transient loss of consciousness. Their BMI was low. One was treated at the emergency outpatient department during the investigation period, but the other two did not consult a medical facility.

Head CT revealed no clear abnormality in the 16 wrestlers who consented, and no neurological deficit was noted in any of the wrestlers (Table 1).

Five wrestlers were treated for head trauma at our emergency outpatient clinic during the investigation period (Table 2). Four of the five had sustained head trauma during matches, and the other during practice. One patient with headache had a contusion in the frontal scalp that required suturing. Skull radiography showed no fracture in all five patients. However, CT showed intracranial traumatic changes in two wrestlers. Three wrestlers, two with intracranial traumatic changes on CT and one with transient loss of consciousness, were hospitalized. The injuries in these three wrestlers were caused by strong impact to the occipital region. No persistent, severe neurological deficit was noted in any of the wrestlers throughout the investigation period.

\section{Representative Cases}

Case 14: A 24-year-old trainee developed headache after suffering a blow to the occipital region while 

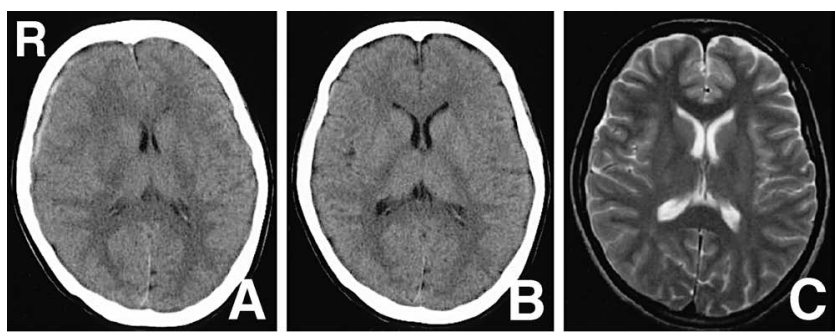

Fig. 1 Case 14. A: Computed tomography (CT) scan showing acute subdural hematoma (ASDH). B: CT scan on the 3rd hospital day showing disappearance of the ASDH. C: $\mathrm{T}_{2}$-weighted magnetic resonance image on the 10th hospital day showing no brain contusion.

practicing a backward defensive fall. Three days later, she received another blow to the occipital region during practice, which induced nausea and exacerbation of headache. She consulted our emergency outpatient clinic. Neurological examination found no abnormality, but CT showed acute subdural hematoma (ASDH), so she was immediately hospitalized (Fig. 1A). She was treated conservatively, and the headache and nausea resolved on the 3rd hospital day, when serial CT confirmed disappearance of the ASDH (Fig. 1B). Magnetic resonance (MR) imaging performed on the 10th hospital day showed no brain contusion (Fig. 1C), and the patient was discharged, walking normally, on the 16th hospital day. A conference with the female professional wrestling organization recommended that she retire from professional wrestling. Case 9: A 23-year-old wrestler with a 5-year career was hit in the occipital region many times during a match, and suffered persistent misty vision immediately after the match. Two days later, limb weakness occurred suddenly during another match, and she became unable to stand. She also had limb dysesthesia, headache, and nausea, so she was transported to our emergency outpatient clinic. On admission, CT showed marked narrowing of the lateral ventricles and cerebral sulci. The diagnosis was diffuse brain swelling (Fig. 2), and she was immediately hospitalized. Conservative treatment using a hypertonic diuretic alleviated the symptoms, and she was discharged, walking normally, on the 10th hospital day.

\section{Discussion}

The frequency of head trauma varies depending on the sport. In our study, four wrestlers sustained head trauma that required treatment at the emergency

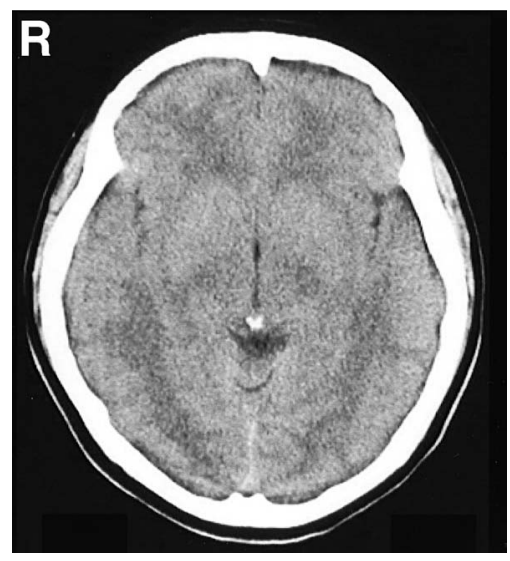

Fig. 2 Case 9. Computed tomography scan showing marked narrowing of the lateral ventricles and cerebral sulci.

outpatient clinic in 817 matches, giving an injury rate per match of $0.5 \%$. The frequency of head trauma per match is $5.1 \%$ (91 cases/1,770 matches) in Shotokan karate (U.K.), ${ }^{6}$ 22.5\% (96/427) in professional boxing (Australia), ${ }^{24)}$ and $5.7 \%(197 / 3,481$ ) in professional kick boxing (Australia). ${ }^{25}$ Compared with these values, the frequency of head trauma per match in our female wrestlers was low, but only 13 regular wrestlers had 817 matches in 14 months, so the number of matches fought per wrestler was clearly higher than in other martial arts. Also, only one of the three wrestlers, who developed retrograde amnesia immediately after head trauma, consulted a medical facility. Therefore, the frequency of all injuries, including mild head injuries, is difficult to accurately determine in female professional wrestlers, and the true head injury rate per wrestler may be much higher.

In this study, female wrestlers with perceived chronic symptoms tended to have a long career in professional wrestling. Boxing encephalopathy is an important condition caused by repeated head trauma, ${ }^{14,18)}$ and the severity correlates with the length of a boxer's career and total number of bouts. ${ }^{8)}$ This study also suggested that repeated head trauma over a long period contributes to the development of chronic symptoms. As diffusion-weighted MR imaging is useful for the evaluation of brain microstructure damage in boxing encephalopathy, ${ }^{26)}$ closer evaluation using MR imaging is considered to be necessary in female professional wrestlers with a long career to evaluate the presence of a condition similar to boxing encephalopathy.

The symptoms observed immediately after head trauma in our subjects included retrograde amnesia, and all wrestlers who had retrograde amnesia had 
sustained head trauma two or more times. Also, these wrestlers had low BMIs among female professional wrestlers. Generally, the incidence of traumas of the whole body is higher in athletes with a high BMI. ${ }^{7)}$ In wrestling, the incidence of catastrophic injuries in the head and neck is higher in light- to middle-weight wrestlers. ${ }^{1)}$ Low BMI indicates low weight relative to height. A light wrestler is more likely to be thrown by opponents, and a tall wrestler is more likely to hit the head against the mat when thrown backwards. Due to these conditions, severe head trauma that may cause retrograde amnesia may be likely to occur in wrestlers with a low BMI.

The five wrestlers treated at our emergency outpatient clinic showed no particular tendency in duration of wrestling career or BMI. However, all three wrestlers who were hospitalized had sustained hard impacts to the occipital region, and one developed ASDH not associated with brain contusion. For anatomical reasons, a blow to the occipital region is considered likely to cause marked rotation of the brain and resultant subdural hematoma. ${ }^{23)}$ Such a mechanism is considered likely in these wrestlers. Case 9, who was hospitalized, had diffuse brain swelling on head CT after sustaining repeated head trauma over a short period. We previously reported that repeated mild impact, which did not cause brain contusion, caused diffuse interstitial edema in the rat brain. ${ }^{9)}$ Professional wrestlers, who are likely to sustain repeated head trauma over a short period, should be considered likely to suffer diffuse brain swelling, and this condition may be characteristic of head trauma in professional wrestlers.

This study showed that neurological symptoms such as retrograde amnesia and transient loss of consciousness caused by head trauma frequently occur in female professional wrestling. However, no medical regulations concerning the entry of wrestlers in matches have been established. The Japanese Boxing Commission has a regulation which forbids a boxer who has been knocked out to participate in a match for at least 90 days $^{21)}$ in consideration of the risk of second impact syndrome, or the exacerbation of brain concussion by additional head trauma. ${ }^{4)}$ There are a few guidelines concerning the safe period to abstain from competition after transient loss of consciousness or post-traumatic amnesia after head trauma sustained during sports activities, $3,5,10-12,17)$ but the absence of a concrete medical basis has lead to criticism, and no definitive guidelines have been established. ${ }^{22)}$ Guidelines based on medical evidence may be prepared through comprehensive evaluation according to common criteria such as the Standardized Assessment of
Concussion $^{15)}$ as proposed by the American Academy of Neurology.

Our Case 14, who developed ASDH and was discharged without sequelae, was later recommended to retire from competition after consultation with the organization. In boxing, which has more deaths due to ASDH than professional wrestling in Japan, Article 39 of the Boxing Commission Rules stipulates "Loss of consciousness during a match and consequent hospitalization may be reasons for recommending retirement to boxers," but there is no mention of the relationship between retirement and ASDH. Case 14 was injured during practice, and she did not lose consciousness (interpreted as loss or severe disturbance of consciousness), so her condition was not a reason for recommending retirement in the strict sense according to the Boxing Commission Rules. However, a male wrestler, who had developed slight ASDH and was treated conservatively, suffered recurrence of ASDH in another match a few months later and died. ${ }^{20}$ ) Therefore, wrestlers with a history of ASDH should be advised to retire because of the risk of recurrence, and periodic medical checks are considered necessary for objective evaluation of their clinical history.

\section{References}

1) Boden BP, Lin W, Young M, Mueller FO: Catastrophic injuries in wrestlers. Am J Sports Med 30: 791-795, 2002

2) Burke DT, Barfoot K, Bryant S, Schneider JC, Kim HJ, Levin G: Effect of implementation of safety measures in tae kwon do competition. Br J Sports Med 37: 401-404, 2003

3) Cantu RC: Guidelines for return to contact sports after cerebral concussion. Phys Sportsmed 14: 75-83, 1986

4) Cantu RC: Second-impact syndrome. Clin Sports Med 17: 37-44, 1998

5) Cantu RC: Return to play guidelines after a head injury. Clin Sports Med 17: 45-60, 1998

6) Critchley GR, Mannion S, Meredith C: Injury rates in Shotokan karate. Br J Sports Med 33: 174-177, 1999

7) Dane S, Can S, Karsan O: Relations of body mass index, body fat, and power of various muscles to sport injuries. Percept Mot Skills 95: 329-334, 2002

8) Guterman A, Smith RW: Neurological sequelae of boxing. Sports Med 4: 194-210, 1987

9) Haga D, Seiki Y, Aikawa H, Honda M, Fukui K, Kano T, Otsuka T, Oishi H, Sugo N: The studies of histological change in rat brain tissue and the neuropsychological change of rat after continuous brain concussion. Neurotrauma Research 15: 45-49, 2003

10) Jordan B, Tsaris P, Warren R (eds): Sports Neurology, ed 2. Philadelphia, Lippincott-Raven Publishers, 1998, p 277 
11) Kelly JP, Nichols JS, Filley CM, Lillehei KO, Rubinstein D, Kleinschmidt-De Masters BK: Concussion in sports. Guidelines for the prevention of catastrophic outcome. JAMA 266: 2867-2869, 1991

12) Kelly JP, Rosenberg JH: The development of guidelines for the management of concussion in sports. $J$ Head Trauma Rehabil 13: 53-65, 1998

13) Leclerc S, Herrera CD: Sport medicine and the ethics of boxing. Br J Sports Med 33: 426-429, 1999

14) Mawdsley C, Ferguson FR: Neurological disease in boxers. Lancet 186: 799-801, 1963

15) McCrea M: Standardized mental status testing on the sideline after sport-related concussion. J Athl Train 36: 274-279, 2001

16) McCrea M, Kelly JP, Randolph C, Cisler R, Berger L: Immediate neurocognitive effects of concussion. Neurosurgery 50: 1032-1042, 2002

17) McCrory P: When to retire after concussion? Br J Sports Med 35: 380-382, 2001

18) McCrory P: Boxing and the brain. Revisiting chronic traumatic encephalopathy. Br J Sports Med 36: 2, 2002

19) Nakaguchi H, Fujimaki T, Ueki K, Takahashi $M$, Yoshida H, Kirino T: Snowboard head injury: prospective study in Chino, Nagano, for two seasons from 1995 to 1997. J Trauma 46: 1066-1069, 1999

20) [News of Fukuda Masakazu's death]. Nikkan Sports News 2000/4/20; sect 1, col 1. Available from: http://ww w.nikkansports.com/jinji/2000/ seikyo000420.html (Jpn)

21) Ohhashi G, Tani S, Murakami S, Kamio M, Abe T, Ohtuki J: Problems in health management of professional boxers in Japan. Br J Sports Med 36: 346-353, 2002

22) Oliaro S, Anderson S, Hooker D: Management of cerebral concussion in sports: the athletic trainer's perspective. J Athl Train 36: 257-262, 2001

23) Pudentz RH, Shelden $\mathrm{CH}$ : The lucite calvarium - a method for direct observation of the brain. J Neurosurg 3: 487-505, 1946

24) Zazryn TR, Finch CF, McCrory P: A 16 year study of injuries to professional boxers in the state of Victoria, Australia. Br J Sports Med 37: 321-324, 2003

25) Zazryn TR, Finch CF, McCrory P: A 16 year study of injuries to professional kickboxers in the state of Victoria, Australia. Br J Sports Med 37: 448-451, 2003

26) Zhang L, Ravdin LD, Relkin N, Zimmerman RD, Jordan B, Lathan WE, Ulug AM: Increased diffusion in the brain of professional boxers: a preclinical sign of traumatic brain injury? AJNR Am J Neuroradiol 24: $52-57,2003$

Address reprint requests to: Jun Nomoto, M.D., Department of Neurosurgery, Toho University, Omori Medical Center, 6-11-1 Omori-nishi, Ota-ku, Tokyo 143-0015, Japan.

\section{Commentary}

Dr. Nomoto and his collaborators chose to investigate an often neglected aspect of sports, head injury. Unlike boxing, where the loss of cerebral function, the knock out, is the declared object of the game, wrestling is obviously only associated with a head injury as an unwanted side effect. The frequency of head injury in professional female wrestlers, however, is high as reported by the authors. Neurological symptoms such as amnesia and loss of consciousness are frequent findings in wrestling.

Conclusions from this report may be of practical relevance: A high body mass index may protect the wrestler from head injury to some degree. Given the exponentially increased risk of permanent brain damage after repeated head injury, as supported by this report, it should certainly prompt professional wrestlers after suffering a head injury to be barred from further matches for a defined interval. Neurosurgeons are most conscious of the devastating effect of permanent brain damage. We should therefore speak up loudly and question the rationale of exposing young humans to the risk of brain injury while in the meantime in most parts of the world it is considered immoral to inflict pain or injuries even upon animals. This report gives good reason to encourage us to pose such questions.

Raimund FIRSCHING, M.D., L.R.C.P., M.R.C.S. Klinik für Neurochirurgie Otto-von-Guericke-Universität Magdeburg, Germany

This paper describes the results of medical examinations for head trauma including body mass index, chronic and acute neurological symptoms, and CT findings in 18 female professional wrestlers. Neurological findings in five wrestlers treated for head trauma during the observation period were also evaluated. Although the author examined the brain with MR imaging only in two patients, diffusionweighted MR imaging and EEG may be useful to identify the microstructural abnormalities and functional damages in the brain. Head injury in female professional wrestlers is a rare issue in neurotraumatology. However, this is not only an acute problem but also an issue for their entire life. Therefore, it is necessary to establish guidelines for monitoring head trauma in female professional wrestlers. This is an important manuscript that cautions of the risk of head injury in female professional wrestling.

Seigo NAGAO, M.D.

Department of Neurological Surgery

Faculty of Medicine

Kagawa University 
Kagawa, Japan

This is an interesting paper which discussed the symptoms related with "head trauma" in female professional wrestlers and we believe it is the first paper specialized in the head injury of female but not male wrestlers. However, there are some questions confusing us, which should be further clarified, in the further study:

1) Why did the author focus on the female but not the male athletes? Are there any significant differences between female and male wrestlers in head trauma? 2) Most common posttraumatic symptoms are cognitive disturbance, memory impairment, emotional disorder etc., which always happened 6 months after head trauma, whereas patients suffering brain concussion are frequently associated with persistent emotional disturbance. However, this paper did not describe any cognitive disturbance or emotional problems even though some patients could be diagnosed as brain concussion based on their symptoms after head trauma such as Case 12.

3) The determination of "chronic symptoms" in this paper is equivocal because the author did not describe when these symptoms happened and how long they persisted. Since most posttraumatic symptoms happened after 6 months, a long-term follow up as long as 1-2 years is really needed to confirm if the symptoms are important.

Shuyuan YANG, M.D. Department of Neurosurgery Tianjin Medical University Hospital Tianjin, P.R.C. 